\title{
Ion Scattering Spectroscopy Intensities for Supported Nanoparticles: the Hemispherical Cap Model
}

\author{
Charles T. Campbell* and Trevor E. James \\ Department of Chemistry \\ University of Washington
}

Seattle, Washington 98195-1700, USA

\begin{abstract}
Nanoparticles of one element or compound dispersed across the surface of another substance form the basis for many materials of great technological importance, like catalysts, fuel cells, sensors and biomaterials. Nanoparticles also often grow during thin film deposition. The size and number density of such nanoparticles are important, often estimated with electron or scanning tunneling microscopies. However, these are slow and often unavailable with sufficient resolution for particles near $1 \mathrm{~nm}$. Because the probe depth of low-energy ion scattering spectroscopy (LEIS) with $\mathrm{He}^{+}$and $\mathrm{Ne}^{+}$is so shallow (less than one atom), it provides quantitative information on the fraction of the surface that is covered by such nanoparticles. Combined with the total amount per unit area, this fraction provides the average particle thickness. When the ions are incident or detected at some angle away from the surface normal, macroscopic screening effects cause interpretation of LEIS signals in terms of area fraction covered to be complicated. In this paper, we report a geometric analysis of particles with the shape of hemispherical caps so that LEIS signals obtained in any measurement geometry can also be used to quantitatively determine the area fraction, average particle thickness and diameter, or number density of particles.

* Corresponding author: email = charliec@uw.edu
\end{abstract}

(C) 2015. This manuscript version is made available under the Elsevier user license http://www.elsevier.com/open-access/userlicense/1.0/ 
A very common structural motif in materials science involves nanoparticles of one material dispersed across the surface of another. Such structures are commonly encountered when making and/or studying catalysts, microelectronics, plasmonic and optical devices, nanoelectrode arrays, sensors, coatings and thin film growth by vapor deposition or other condensation processes. For example, metal nanoparticles dispersed across the surfaces of oxides and carbon supports are key ingredients in many heterogeneous catalysts, fuel cells, other electrocatalysts and photocatalysts, all of which are crucial for energy, fuel and environmental technologies and chemical processing. One of the three growth modes that occur during thin film growth by vapor deposition is the Volmer-Weber mode, whereby clusters of the deposited material first nucleate and then grow as 3D nanoparticles on the substrate.[1-7]

A method to estimate the size and number density of such nanoparticles is based upon measurements of the intensities of peaks associated with substrate and adsorbate elements using low-energy ion scattering spectroscopy, LEIS. A model that has been applied for analyzing these LEIS intensities is the hemispherical cap model introduced by Diebold et al.,[8] which assumes that the nanoparticles all have the shape of hemispheres and the same diameter. It is often applied to cases where the LEIS intensities of substrate and adsorbate peaks have been measured versus the amount of adsorbate deposited on the flat substrate surface, in which case the further assumption is made that the number of particles per unit area remains constant. This assumption is based on the well-known fact that in many such systems, the number density of particles increases in the first few percent of a monolayer (ML), but quickly reaches the so-called saturation density, after which it stays nearly constant until very high coverages.[2] This number density is estimated from a fit to these plots of intensities versus coverage (or the intensities at any one coverage) to the resulting equations of this hemispherical cap model, which also gives the average diameter of the particles at each coverage.[8] Unfortunately, those equations for the hemispherical cap model only were intended to apply to one measurement geometry (when the ions are incident $45^{\circ}$ from normal and the scattered ion intensities have been measured for a take-off angle normal to the substrate surface). Unfortunately, there is an error in that equation. Here, we present a correction and extension of that model that allows it to be applied at any angles of incidence and detection. Other angles are often demanded by the constraints on sample mounting or manipulation imposed by the particular vacuum chamber being used. 
Here we consider LEIS using noble gas ions like $\mathrm{He}^{+}$at energies below $2 \mathrm{kV}$. Since such ions get neutralized with essentially unit probability when they penetrate the electron density of a solid by an amount deeper than a small fraction of an atom, the ions that survive scattering from the solid and get detected as LEIS signal probe only the topmost atomic layer of the solid.[9] Let us define here more closely what we mean by "topmost atomic layer" here. We do not mean the topmost plane of atoms, since substantial signal comes from the $2^{\text {nd }}$ plane of atoms on very open crystal faces. For example, the atoms within the very open topmost plane of both the O- and $\mathrm{Zn}$ terminated faces of $\mathrm{ZnO}(0001)-\mathrm{O}$ and $\mathrm{ZnO}(000-1)-\mathrm{Zn}$ are separated by a large distance $(0.33$ $\mathrm{nm}$ ), but their two topmost atomic planes are nearly coplanar, separated by only $0.06 \mathrm{~nm}$. Therefore, both these top two planes give signals in $\mathrm{He}^{+}$LEIS. Still, the $\mathrm{Zn} / \mathrm{O}$ signal ratio is 2.5to 5-fold larger on the $\mathrm{Zn}$ - face than the $\mathrm{O}$ - face for 500 to $700 \mathrm{eV}$ incident ions.[10] This is similar to what one would expect from what one "sees" upon visual inspection of a crystal model of these $\mathrm{ZnO}$ faces made using touching spheres to represent the atoms. This visual model is thus close to what is seen in LEIS signals using noble gas ions like $\mathrm{He}^{+}$at energies below $2 \mathrm{kV}$. Similarly, essentially all the LEIS signal comes from the topmost atomic plane when it is closepacked[9], as would again be expected from such a visual model. We do not consider here spectroscopies where neutrals are detected, since they can probe much deeper.

We consider here systems where the adsorbate $A$ is on a flat substrate surface $S$ in the form of islands, and assume that the ions have a constant neutralization probability when they strike area that are material S and another, perhaps different neutralization probability when they strike areas covered by A islands.

We first consider the case where the adsorbate is in the form of single atom-thick $2 \mathrm{D}$ islands. In this case, the signal intensity for the LEIS peak for the adsorbate, $\mathrm{I}_{\mathrm{A}}$, is proportional to the fraction of the surface physically covered by islands of $A, f_{A}$ :

$$
\mathrm{I}_{\mathrm{A}}=\mathrm{f}_{\mathrm{A}} \mathrm{I}_{\mathrm{A}}^{0}
$$

where $\mathrm{I}_{\mathrm{A}}{ }^{0}$ is the signal when $100 \%$ of the surface is covered by a $2 \mathrm{D}$ layer of $\mathrm{A}$. The substrate peak's intensity, $\mathrm{I}_{\mathrm{S}}$, is just:

$$
\mathrm{I}_{\mathrm{S}}=\left(1-\mathrm{f}_{\mathrm{A}}\right) \mathrm{I}_{\mathrm{S}}^{0}
$$


where $\mathrm{I}_{\mathrm{S}}{ }^{0}$ is its signal when none of the surface is covered by A (i.e., from a clean $\mathrm{S}$ surface). Here we assume that since the islands are only one atom thick, any shadowing of uncovered substrate by the island edges is negligible at any angles of incident ions or detection. This is certainly true for large islands, but may fail when the islands are only a few atoms across.

When the adsorbate is in the form of uniform-size 3D hemispherical nanoparticles instead of single atom-thick 2D islands, the same analysis as above applies if the ions are both incident and detected normal to the surface. Thus, Eqs. (1) and (2) still apply, except that $\mathrm{I}_{\mathrm{A}}{ }^{0}$ now refers instead to the signal when $100 \%$ of the surface is covered by 3D islands of A. This $100 \%$ coverage is impossible to realize experimentally while maintaining the shape of hemispherical caps, so $\mathrm{I}_{\mathrm{A}}{ }^{0}$ is usually approximated experimentally when studying film growth as the signal when $100 \%$ of the surface is covered by a 3D layer of A. This is best measured after having added just enough $\mathrm{A}$ to the surface such that its $3 \mathrm{D}$ islands have grown together to mask all of the signal from $\mathrm{S}$, which most closely approximates the same surface roughness factor of hemispherical caps. It is important to have the same roughness (i.e., the same distribution of local angles of the surface of A relative to the substrate surface normal) because the number of $\mathrm{A}$ surface atoms per unit projected area of A onto the planar substrate varies inversely with the cosine of the local angle of the surface of A relative to the substrate surface normal. Thus, the LEIS signal for A per unit projected area of A onto the planar substrate probably also depends on that local angle. Therefore, this rough surface of pure A with a very similar distribution of surface angles as for hemispherical caps is a better reference signal for estimating $\mathrm{I}_{\mathrm{A}}{ }^{0}$ than a flat surface of bulk A. It is also often easier to achieve this experimentally than a flat surface of pure A on the same day as the nanoparticle measurements, unless one has the ability to mount a separate reference sample in the same analysis position. Unless the A atoms are quite mobile, this surface roughness persists or even gets worse as film thickness increases. If A does not like to wet the substrate, it can also get rougher and can even dewet upon annealing.

When the ions are incident or detected at some angle away from the substrate surface normal, the analysis is more complex, since the 3D hemispheres mask more of the solid than their actual footprint (or interfacial contact area on S). Consider first when ions are incident still normal to the surface but detected at some polar angle $\theta_{\mathrm{d}}$ from normal, as shown in Fig. 1 for a particle of radius $r$. As shown, more of the surface is masked by the particle than its footprint 
area $\pi r^{2}$. The left side masked area is now half of an ellipse with semi-minor axis of length $r$ and semi-major axis of length $b=r / \cos \theta_{d}$. Since the area of the full ellipse would be $\pi \mathrm{rb}=$ $\pi \mathrm{r}^{2} / \cos \theta_{\mathrm{d}}$, the masked area by one particle is now:

$$
\text { Area per particle }=\pi \mathrm{r}^{2}\left(1+1 / \cos \theta_{\mathrm{d}}\right) / 2 \text {. }
$$

If there is a number density of $\mathrm{n}$ such particles per unit area and their separations are large enough that their shadows do not overlap, the fraction of the substrate's area that is masked by the whole collection of particles of $\mathrm{A}$ is just:

$$
\mathrm{f}_{\mathrm{A}}=\mathrm{n} \times \text { Area per particle }=\mathrm{n} \pi \mathrm{r}^{2}\left(1+1 / \cos \theta_{\mathrm{d}}\right) / 2
$$

When the angle of detection is normal to the surface $\left(0^{\circ}\right)$ but the ions are incident at some angle $\theta_{\mathrm{i}}$ from normal, one gets a nearly identical equation for the fraction masked by A:

$$
\mathrm{f}_{\mathrm{A}}=\mathrm{n} \pi \mathrm{r}^{2}\left(1+1 / \cos \theta_{\mathrm{i}}\right) / 2
$$

Finally, when the ions are incident at some angle $\theta_{\mathrm{i}}$ from normal and the angle of detection is at some angle $\theta_{\mathrm{d}}$ from normal, and these two polar angle directions are separated by $180^{\circ}$ in the azimuthal angle, the fraction of substrate $\mathrm{S}$ masked by $\mathrm{A}$ is:

$$
\mathrm{f}_{\mathrm{A}}=\mathrm{n} \pi \mathrm{r}^{2}\left(1 / \cos \theta_{\mathrm{i}}+1 / \cos \theta_{\mathrm{d}}\right) / 2
$$

It also has the proper limits when either $\theta_{\mathrm{i}}$ or $\theta_{\mathrm{d}}$, or both, is decreased to zero (normal). It is still a pretty good approximation even when the azimuthal separation decreases to $90^{\circ}$. Using Eq. (6) for $\mathrm{f}_{\mathrm{A}}$ allows one to still model the intensities of the adsorbate and substrate LEIS peaks using Eqs. (1) and (2), respectively. As noted above, $\mathrm{I}_{\mathrm{A}}{ }^{0}$ must now refer to the signal when $100 \%$ of the surface is covered by a 3D islands of A, estimated experimentally as described above.

To understand why this $\mathrm{I}_{\mathrm{A}}{ }^{0}$ is the appropriate reference signal for use when combining Eq. (1) with Eq. (6), it is useful to imagine the situation when the A particles are packed close enough together such that the areas masked by each particle just start to overlap, as shown schematically in Fig. 2. One must actually imagine this in more than one dimension, with the hypothetical situation where fraction of substrate $S$ masked by $A, f_{A}=n \pi r^{2}\left(1 / \cos \theta_{i}+\right.$ $\left.1 / \cos \theta_{\mathrm{d}}\right) / 2$, just reaches 1.0 . If the particles were packed any closer than this, no extra signal for 
A would be gained, since parts of each particle would then move into regions shadowed by its neighbors. We thus assume that the signal for A would remain the same with further increases in packing density right up to the limit where the $3 \mathrm{D}$ islands of $\mathrm{A}$ have grown together to mask all of the signal from substrate $S$, which we defined above as giving $\mathrm{I}_{\mathrm{A}}{ }^{0}$ as its signal for $\mathrm{A}$.

As noted above, Diebold et al.[8] introduced an identical model for analyzing these LEIS intensities for adsorbates in the form of hemispherical caps. They analyzed only the special case when the angle of detection is normal to the surface $\left(\theta_{d}=0^{\circ}\right)$ and the ions are incident at $\theta_{i}=45^{\circ}$ from normal. For the fraction masked by A, they used that $f_{A}=3 n \pi r^{2} / 2=(1.5) n \pi r^{2}$. However, the true value for this geometry is $f_{A}=(1.207) n \pi r^{2}$, obtained using either Eq. (5) or (6). It is possible that they stated incorrectly that $\theta_{i}=45^{\circ}$ in the paper, since $\theta_{i}=60^{\circ}$ would give their result.

To demonstrate how Eq. (6) is used, the growth of vapor-deposited $\mathrm{Cu}$ onto slightly reduced $\mathrm{CeO}_{2}(111)$ was investigated using $\mathrm{He}^{+}$LEIS at 300K, as shown in Fig. 3(a). The ions are incident $45^{\circ}$ from surface normal at an energy of $1.5 \mathrm{keV}$ and detected normal to the surface here. (The other experimental details and analysis of this same system with other surface analysis techniques is fully presented elsewhere[11]). The integrated intensity of the Ce signal was normalized to the integrated $\mathrm{Ce}$ area with no $\mathrm{Cu}$, and the $\mathrm{Cu}$ signal was normalized to a thick $\mathrm{Cu}$ overlayer that completely masked the substrate's Ce LEIS signal. When normalized in this way, the $\mathrm{Cu}$ signal is a direct measure of the fraction of the ceria surface masked by $\mathrm{Cu}$ particles and the Ce signal measures the fraction that is not masked. The Cu LEIS signal increases with $\mathrm{Cu}$ coverage and the $\mathrm{Ce}$ signal decreases for all $\mathrm{Cu}$ coverages, indicating $\mathrm{Cu}$ does not diffuse into the substrate, but rather forms a surface layer on $\mathrm{CeO}_{1.95}(111)$. The data in Fig. 3(a) are compared to the layer-by-layer model and the hemispherical cap model. The data deviate markedly from layer-by-layer growth but are well fit using the hemispherical cap model described above. This number density is the only fitting parameter, since it alone determines the value of the particle diameter $(\mathrm{D}=2 \mathrm{r})$ at any given total $\mathrm{Cu}$ coverage. It was determined for the data in Fig. 3a from the best fit to Eqs. (1) and (2) (with Eq. (6) for $\mathrm{f}_{\mathrm{A}}$ ) to the data up to a coverage of 2.2 ML (above which the masked area of neighboring particles may start to overlap significantly and the model would fail). This best fit was generated using a least squares analysis. It gave a $\mathrm{Cu} 3 \mathrm{D}$ particle density of $7.8 \times 10^{12}$ particles $/ \mathrm{cm}^{2}$. 
Up to the fit coverage of $2.2 \mathrm{ML}$, the RMS error of the data relative to this fit is 0.021 (in the same units as the $y$-axis here). If we used this same number density with the original equation from Ref.[8], the RMS error increases to0.069, 3-fold larger.

The normalized LEIS signals in Fig. 3(a) directly provide the fraction of the ceria surface whose signal is masked by $\mathrm{Cu}$ particles, and the fraction of the total maximum $\mathrm{Cu}$ signal (due to complete coverage by $\mathrm{Cu}$ ) that is observed at any given $\mathrm{Cu}$ coverage. If we assume the actual footprint of the $\mathrm{Cu}$ particles is expanded due to macroscopic shadowing by the same factor as is the case for hemispherical caps at this LEIS measurement geometry $\left(\left(1+1 / \cos \theta_{\mathrm{i}}\right) / 2=1.207\right.$, see Eq. (5) or (6)), we can divide these fractions by 1.207 to estimate the area fractions actually covered by $\mathrm{Cu}$ particles, and not just masked by them from ion trajectories. Since we know the average $\mathrm{Cu}$ film thickness from the $\mathrm{Cu}$ coverage (assuming the $\mathrm{Cu}$ particles have the density of bulk $\mathrm{Cu}$ (solid)), and we can divide it by this covered fraction for each LEIS data point (both $\mathrm{Cu}$ and $\mathrm{Ce}$ ) to get the average particle thickness. Fig. 3(b) shows the average $\mathrm{Cu}$ particle thickness versus $\mathrm{Cu}$ coverage for both ceria films estimated in this way from each LEIS data point. Since the average thickness of a hemispherical cap is $1 / 3$ its diameter, we have multiplied this thickness by 3 to show the corresponding average diameter along the right-hand axis here. For comparison, we show the result expected from the same hemispherical-cap model and particle number density as used for the best fit to the top part of Fig. 3(a). The agreement is excellent.

The hemispherical cap model was also introduced by Diebold et al.[8] for analyzing electron spectroscopy signals, where they derived equations for the intensities of peaks associated with substrate and adsorbate elements. It has been widely applied for analyzing signals in Xray photoelectron spectroscopy or Auger electron spectroscopy during film growth where 3D particles are formed. However, those equations only apply when the electron intensities have been measured for a take-off angle normal to the substrate surface. We recently presented an extension of that model that allows it to be applied at other angles of electron detection.[12] We have found that when electron spectroscopy is used together with LEIS to analyze film growth, it provides very powerful information on film morphology.[5, 11, 13, 14]

\section{Acknowledgements}


The authors thank Stephanie Hemmingson for help with data acquisition, and her and Gabe Feeley for insightful discussions. They gratefully acknowledge financial support for this work by the U.S. Department of Energy-Office of Basic Energy Sciences, Chemical Sciences Division under grant no. DE-FG02-96ER14630.

\section{References}

[1] C. Argile, G.E. Rhead, Adsorbed Layer and Thin-Film Growth Modes Monitored by Auger-Electron Spectroscopy, Surface Science Reports, 10 (1989) 277-356.

[2] J.A. Venables, Atomic Processes in Crystal Growth, Surface Science, 299 (1994) 798-817.

[3] C.T. Campbell, Ultrathin metal films and particles on oxide surfaces: Structural, electronic and chemisorptive properties, Surface Science Reports, 27 (1997) 1-111.

[4] J.A. Farmer, C.T. Campbell, Ceria Maintains Smaller Metal Catalyst Particles by Strong MetalSupport Bonding, Science, 329 (2010) 933-936.

[5] J.A. Farmer, J.H. Baricuatro, C.T. Campbell, Ag Adsorption on Reduced $\mathrm{CeO}_{2}(111)$ Thin Films, Journal of Physical Chemistry C, 114 (2010) 17166-17172.

[6] Q. Fu, T. Wagner, Interaction of nanostructured metal overlayers with oxide surfaces, Surface Science Reports, 62 (2007) 431-498.

[7] W.M. Tong, R.S. Williams, Kinetics of surface growth - phenomenology, scaling, and mechanisms of smoothening and roughening, Annual Review of Physical Chemistry, 45 (1994) 401-438.

[8] U. Diebold, J.M. Pan, T.E. Madey, Growth Mode of Ultrathin Copper Overlayers on $\mathrm{TiO}_{2}(110)$, Physical Review B, 47 (1993) 3868-3876.

[9] H.H. Brongersma, M. Draxler, M. de Ridder, P. Bauer, Surface composition analysis by low-energy ion scattering, Surface Science Reports, 62 (2007) 63-109.

[10] J. Yoshihara, J.M. Campbell, C.T. Campbell, Cu films on a Zn-terminated ZnO(0001) surface: structure and electronic properties, Surface Science, 406 (1998) 235-245.

[11] T.E. James, S.L. Hemmingson, T. Ito, C.T. Campbell, The Energetics of Cu Adsorption and Adhesion onto Reduced $\mathrm{CeO}_{2}(111)$ Surfaces by Calorimetry, Submitted.

[12] J.C. Sharp, C.T. Campbell, Quantitative modeling of electron spectroscopy intensities for supported nanoparticles: The hemispherical cap model for non-normal detection, Surface Science, 632 (2015) L5L8.

[13] J. Zhu, J.A. Farmer, N. Ruzycki, L. Xu, C.T. Campbell, G. Henkelman, Calcium adsorption on $\mathrm{MgO}(100)$ : Energetics, structure, and role of defects, Journal of the American Chemical Society, 130 (2008) 2314-2322.

[14] J.C. Sharp, Y.X. Yao, C.T. Campbell, Silver Nanoparticles on $\mathrm{Fe}_{3} \mathrm{O}_{4}(111)$ : Energetics by $\mathrm{Ag}$ Adsorption Calorimetry and Structure by Surface Spectroscopies, Journal of Physical Chemistry C, 117 (2013) 24932-24936. 


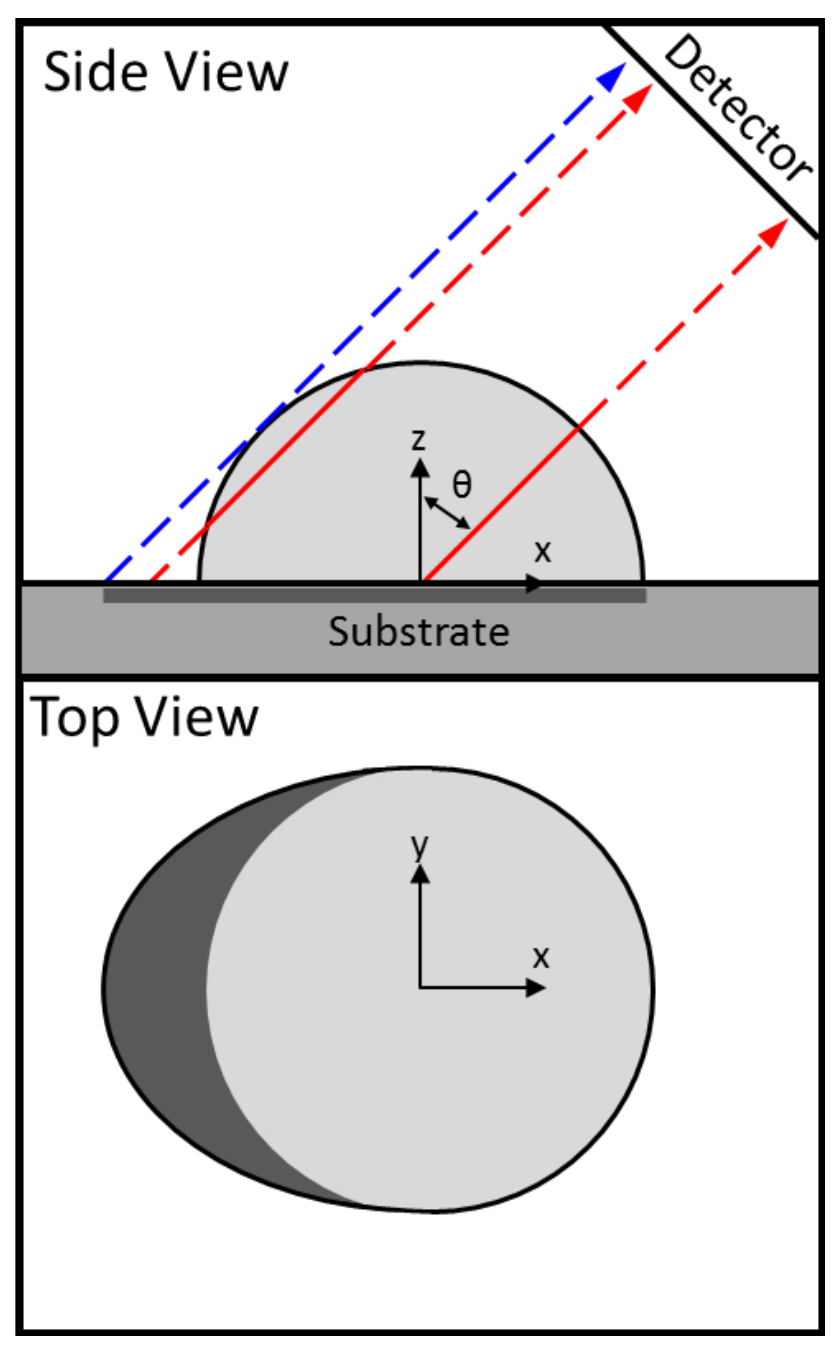

Figure 1. Schematic diagram of a hemispherical cap of adsorbate and the underlying substrate from both side and top views. The detector is at the angle shown but infinitely far away compared to the particle size. The y axis (not shown) is out of plane. Ions scattered from the substrate will be completely blocked from reaching the detector by the hemispherical cap if they originate from the shadow created by the cap (darker shaded area). The hashed area of the substrate therefore represents the effective spectroscopic footprint of the particle at this particular angle of detection ( $\theta_{\mathrm{d}}$ from normal to the substrate surface). 


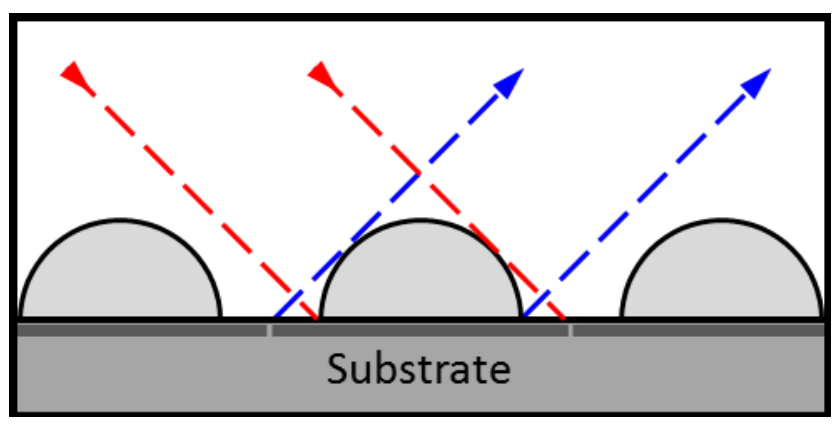

Figure 2. Schematic representation in one dimension of the hypothetical situation wherein hemispherical caps are packed together so closely (in both dimensions) that the fraction of substrate signal masked by A just reaches 1.0. The signal for A gets no larger at higher packing densities, even when A particles touch and cover $100 \%$ of the substrate. 


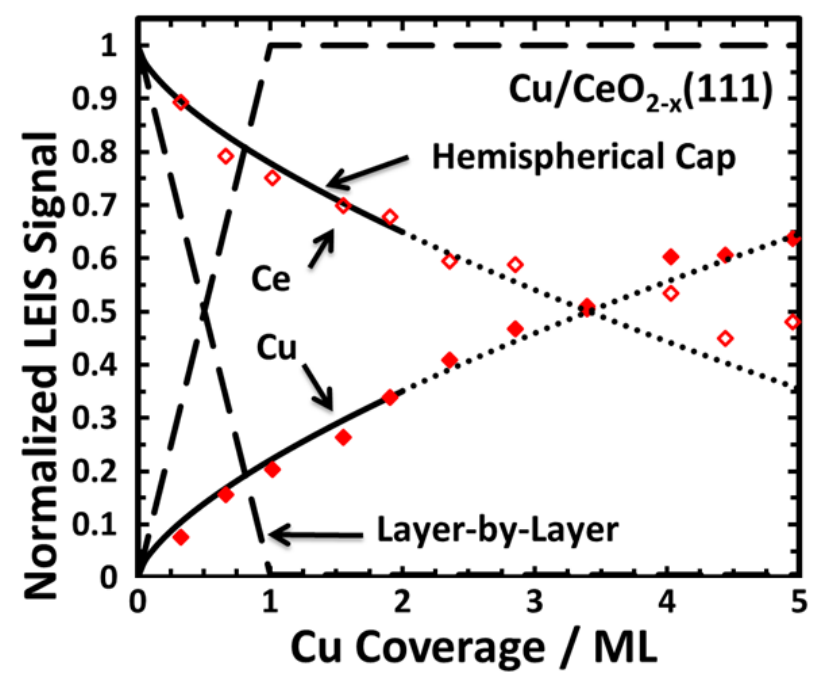

Figure 3(a): Integrated $\mathrm{Cu}$ (closed diamonds) and $\mathrm{Ce}$ (open diamonds) LEIS signal intensities (normalized to that from a thick $\mathrm{Cu}$ film and clean $\mathrm{CeO}_{2-\mathrm{x}}(111)$, respectively) as a function of $\mathrm{Cu}$ coverage after deposition onto $\mathrm{CeO}_{1.95}(111)$ (diamonds) at $300 \mathrm{~K}$. The $\mathrm{He}^{+}$ions are incident $45^{\circ}$ from surface normal at an energy of $1.5 \mathrm{keV}$ and detected normal to the surface. The dashed lines corresponds to the normalized LEIS signals that would be observed if $\mathrm{Cu}$ grew in a layerby-layer fashion, while the solid lines corresponds to the best fit to the hemispherical cap model (Eq. (6)), whereby $\mathrm{Cu}$ grows as hemispherical caps with a fixed radius (at any given coverage) and a fixed particle density at all coverage of $7.8 \times 10^{12}$ particles $/ \mathrm{cm}^{2}$. This model is only reasonable up to $\sim 35 \%$ of the surface being covered by particles, since they may start to overlap with each other at higher coverage, so the dotted lines after that are only a guide to the eye. 


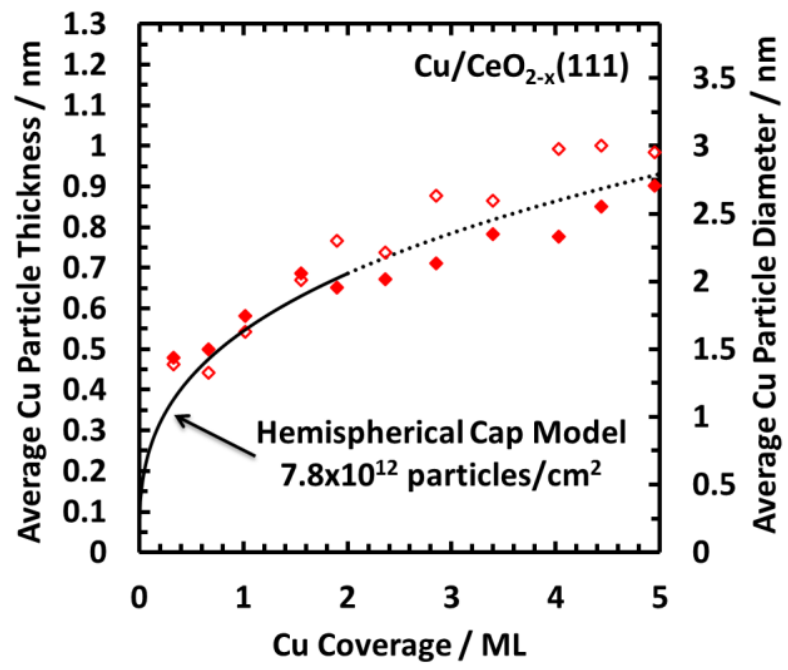

Figure 3(b): The average $\mathrm{Cu}$ particle thickness versus $\mathrm{Cu}$ coverage calculated from the $\mathrm{Cu}$ (closed) and Ce (open) LEIS data points of Fig. 3(a), and on the right axis, the average diameter of hemispherical caps that corresponds to this thickness. Also shown is the result expected for the same hemispherical-cap model and particle number density as used for the best fit to the data in part (a). 